\title{
SIMULACION NUMERICA DE LLUVIAS EN EL NORTE DEL PERU
}

\author{
Mauricio Carrillo ${ }^{\mathrm{a}, \mathrm{b}}, \mathrm{Juan} \mathrm{Bazo}^{\mathrm{b}}$, Octavio Fashéa ${ }^{\mathrm{a}}$, Martín Calvo ${ }^{\mathrm{a}}$, \\ Jaime Torres ${ }^{\mathrm{a}}$, Manuel Sullón ${ }^{\mathrm{a}}$, Walter Molina ${ }^{\mathrm{a}, \mathrm{c}}$ \\ ${ }^{a}$ Laboratorio de Física de Eventos Naturales. Departamento de Física Interdisciplinaria, Facultad de Ciencias \\ Físicas, Universidad Nacional Mayor de San Marcos. Apartado Postal 14-0149, Lima 14, Perú. \\ ${ }^{b}$ Modelamiento Numérico del Tiempo y el Clima. Servicio Nacional de Meteorología e Hidrología \\ 'Servicio de Agua Potable y Alcantarillado de Lima
}

\begin{abstract}
Resumen
Lluvias extremas se han observado en el norte del Perú, el impacto de estas lluvias sobre la infraestructura presente en las ciudades es importante, esto debido a la alta vulnerabilidad de la zona. Los eventos ocurren principalmente en verano. Por lo tanto, se han seleccionado catorce casos de lluvia extrema, las que se registraron durante diciembre y marzo del 2001 y 2002.

En la presente investigación se realiza una evaluación de la simulación numérica de lluvias en el norte del Perú. Se empleo un modelo regional atmosférico llamado RAMS (Regional Atmosphere Modeling System) el cual ha sido implementado en la zona norte del Perú usando la técnica de anidamiento para reducir la resolución. La evaluación de la simulación de lluvias es realizada midiendo la correlación entre las lluvias registradas y las simuladas por el modelo RAMS.

Los resultados muestran una mayor correlación para la cuenca alta, un valor intermedio de correlación para la cuenca media, y los menores valores de correlación se observa en la zona baja de la cuenca. Estos valores para la cuenca alta, media y baja son: $0.49,0.44$, y 0.15 respectivamente. Aunque estos valores son aparentemente bajos, se ha presentado valores de correlación de 0.90 para la cuenca baja de la cuenca, al simular todo el evento de lluvia extrema. También valores de correlación de 0.94 fue observada en la parte alta de la cuenca. Se mencionan algunas explicaciones de los bajos valores de correlación, así como algunas medidas de mejora en los sistemas de adquisición de datos.
\end{abstract}

Palabras Claves: Lluvias Extremas, RAMS, Simulación Numérica, Norte del Perú.

\begin{abstract}
Extreme rains have been observed in the north of Peru, the impact of these rains over the present infrastructure in the cities is important, this due to the high vulnerability of the zone. The events happen mainly in summer. Therefore, fourteen cases of extreme rain have been selected, those that registered during the December and March of 2001 and 2002.

In the present research an evaluation of the numerical rain simulation is made in north of Peru. Use a regional model atmospheric call RAMS (Regional Atmosphere Modeling System). It has been implemented in the North zone of Peru using the nesting technique to reduce the resolution. The evaluation of the rain simulation is made measuring the correlation between registered rains and the simulated ones by the model RAMS.

The results show a greater correlation for the high river basin, an intermediate value of correlation for the average river basin, and the smaller values of correlation are observed in the low zone of the river basin. These values for the high, intermediate and low river basin are: $0.49,0,44$, and 0,15 respectively. Although these values are apparently low, has appeared values of correlation of 0,90 for the low river basin of the river basin, when simulating the entire extreme rain event. Also values of 0,94 correlations were observed in the high part of the river basin. Some explanations of the low values of correlation, as well as some measures of improvement in the systems of data acquisition are mentioned.
\end{abstract}

Keywords: Extreme Rains, RAMS, Numerical Simulation, North of Peru. 


\section{Introducción}

Las lluvias extremas en el norte del Perú, se producen se producen entre los meses de diciembre y abril, durante años El Niño las cantidades de las precipitaciones se incrementan respecto a su variación media. Esto causa grandes pérdidas económicas e impactos sociales; convirtiendo esta zona del Perú en un área altamente vulnerable a estos eventos. Sin embargo, casos de lluvias extremas también ocurren en años no El Niño. La dinámica de lluvias en la parte oeste de Sudamérica, ha sido estudiada anteriormente por varios científicos; de las diferentes teorías, la explicación dada por Horel y Cornejo-Garrido [1] presentan como principal mecanismo de generación de precipitación al sistema de brisas mar-tierra. Además J. Bendix [2] hace una génesis de las brisas mar-continente con ayuda de imágenes AHVRR, adicionando el paso de humedad desde el amazonas a través de los andes el cual enriquece el contenido de humedad en la zona. La presente investigación pretende verificar la habilidad de un modelo numérico de escala regional para simular correctamente lluvias en el norte del Perú. También verificar la importancia de los efectos orográficos en la zona de estudio. Con el resultado de la presente investigación se espera mejorar los sistemas de alerta temprana a fenómenos atmosféricos y medir la predictabilidad de estos eventos. Para facilitar la toma de decisiones bajo impacto de clima extremo.

\section{El Modelo}

El modelo regional usado es el llamado RAMS (Regional Atmosphere Modeling System) versión 4.4. RAMS fue desarrollado por CSU (Colorado State University) y ATMET (Atmospheric, Meteorological, and Environmental Technologies). El concepto de RAMS nació al inicio de la década de los 80's en Colorado State University. Allí en el Departamento de Ciencia Atmosféricas existían tres modelos, el modelo CSU nube/mesoescale [3], una versión hidrostática del modelo de nubes [4], y el modelo brisa de mar descrito por Mahrer y Pielke [5]. El modelo brisa de mar y el modelo nube/mesoescala fueron desarrollados en los inicios de los 70's. Estos modelos se unificaron en un sistema, y el modelo de nubes con la versión hidrostática fue adicionado en 1983, formando la primera versión de RAMS. RAMS es un modelo de predicción numérica multipropósitos diseñado para simular circulación atmosférica en escalas que van de un hemisferio terrestre hasta simulación de largos Eddies de la capa limite. Su aplicación mas frecuente son las simulaciones de fenómenos atmosféricos a mesoescala (escala horizontal de $2 \mathrm{Km}$. a $2000 \mathrm{Km}$.) para propósitos en rangos de pronostico del tiempo operacional a aplicaciones reguladoras de calidad de aire. El predecesor código de RAMS fue desarrollado inicialmente para realizar investigación en áreas de modelamiento de sistemas del tiempo forzadas orográficamente, simulación de nubes convectivas, sistemas convectivos a mesoescala, nubes cirrus, y precipitación en general. Alguna implementación de RAMS involucra una gran variedad de opciones y características para optimizar el modelo en los propósitos específicos. RAMS es equipado con un múltiple equipamiento de grilla, el cual permite a las ecuaciones del modelo ser resueltas simultáneamente en un número de interacciones computacional de diferente resolución espacial. La máxima resolución de grilla es usada para modelar los detalles de sistemas atmosféricos a pequeña escala, como flujos sobre terreno complejo y circulación termal inducida en superficie. La grilla mas gruesa, menor resolución, es usada para modelar los detalles el ambiente de estas pequeños sistemas, así proveer condiciones de frontera a la grilla de región fina. Adicionalmente, la grilla gruesa es usada para simular sistemas atmosféricos a larga escala, el cual interactúa con el sistema a mesoescala resuelto en la grilla mas fina. Varios aspectos de la estructura de RAMS son descritos a continuación.

Estructura de Grilla: La grilla Arakawa $\mathrm{C}$ es empleada [6]. Las variables termodinámicas y de humedad son definidas en el centro de un volumen de grilla con la componente de la velocidad desfasada $1 / 2$ de un espacio de grilla en su dirección normal. Este desfase tiene diversas ventajas incluyendo isotropía de la localización de la componente de velocidad relativa a las variables termodinámicas las cuales son muy 
importantes para la conservación de masa y flujo.

Sistema de Coordenadas Horizontal: La transformación de la grilla horizontal es una proyección estereográfica polar rotada. El polo de la proyección del mapa es rotada para un localización especificada por el usuario, típicamente el centro del anidado grueso del modelo. Esto reduce la cantidad de distorsión entre espaciamiento de la grilla del modelo y la correspondiente distancia sobre la Tierra. También, para pequeños dominios, la estructura horizontal es muy próxima a la coordenada Cartesiana o UTM.

Sistema de Coordenada Vertical: La transformación de coordenada vertical es la paralela al terreno representación de coordenada sigma-z como es descrita por Gal-Chen y Somerville [7]. La coordenada sigma-z tiene la ventaje sobre la coordenada sigma-p en que los niveles del modelo están en la misma altura absoluta sobre en suelo en todos los tiempos durante una simulación.

Condiciones de Frontera Anidadas: RAMS es equipado con un esquema de múltiples grillas anidadas el cual permite a las ecuaciones del modelo ser resueltas simultáneamente en la interacción de la grilla computacional de diferente resolución espacial. La interacción dosvías entre las grillas anidadas es realizada siguiendo el esquema de Clark y Farley [8] y Clark y Hall [9]. La solución dependiente del tiempo del modelo es primero actualizada en la grilla gruesa. Una interpolación tri-cuadrática espacial es después realizada para obtener valores los cuales son asignados a la frontera espacial de la grilla anidada mas fina dentro de una grilla gruesa. Los campos del modelo en una grilla fina son después actualizados usando valores de una grilla gruesa interpolada como las condiciones de frontera espacial. Una vez que la grilla fina esta en el mismo nivel de tiempo como la grilla gruesa, promedios espacial local de los campos de grilla fina son obtenidos y usados para sobreponer los campos de la grilla gruesa. Cuidado es tomado en la implementación del esquema para asegurar que la interpolación y ciclo de promedios es reversible y que la masa y momento es conservado a través de las interfases de las grillas.

Modelo Físico y Numérico: El grupo de ecuaciones mas usadas por RAMS es la ecuación no hidrostática de quasi-Boussinesq descrita por Tripoli y Cotton [3]. Hay ecuaciones pronostico para todos los estados de la variables incluyendo $\mathrm{u}, \mathrm{v}, \mathrm{w}$, temperatura potencial, razón de mezcla y función de Exner.

Formulación de Diferencias Finitas: El modelo es básicamente de segundo orden en tiempo y espacio. Los términos de advección son formulados en un modo de flujo conservativo. Así, masa, momento, y energía son conservados. El modelo también tiene un esquema "timesplit" para manejar términos de onda de sonido. Este esquema calcula los términos responsables para la onda de sonido en un pequeño delta de tiempo más largo que la escala de tiempo de términos como la advección [3].

Modelo de Suelo: Para las condiciones de frontera inferior del modelo de atmósfera, la capa de superficie y parametrizaciones de suelo se describen en detalle por Tremback y Kessler [10] son usados. Se formulan ecuaciones de pronóstico para la temperatura de suelo superficial y contenido de agua asumiendo una capa interfase de profundidad finita suelo/atmósfera. La capa de flujo de calor superficial, momentum, y vapor de agua en el aire es calculado con el esquema de Louis [11]. Este esquema aproxima los perfiles de funciones de Businger et al. [12] con expresiones analíticas. El esquema de Louis también exhibe un comportamiento más real que el esquema de Businger en el límite de convención libre y en condiciones muy estables.

Uso de Suelos: Característica de uso de suelo variable es empleado usando datos digitales de alta resolución de USGS. La entrada distingue entre agua y superficies de suelo, y el ultimo es subdividido en aproximadamente en 18 clases. A cada celda de grilla del modelo le es asignada su predominante tipo de uso de suelo (o porcentaje de suelo, porcentaje de agua donde sea apropiado) al analizar el modo de distribución de tipos dentro de cada celda. Cada celda de grilla es entonces caracterizada por una longitud de rugosidad y albedo. 
Cobertura Vegetativa: La actual parametrización de vegetación es derivada de un trabajo de Avissar [13]. Para cada columna de grilla, una clase de vegetación dominante es definida. Los tipos usados en el modelo BATS son incluidos.

Microfísica y Esquema de Convección: En grillas gruesas RAMS usa un esquema de convección basado en Kuo [14]. En todas las grillas, la explicita parametrización de microfísica puede ser activada.

Radiación: RAMS actualmente contiene dos grupos de esquemas radiactivos, el esquema descrito por Mahrer y Pielke [5] y el esquema descrito por Chen y Cotton [15]. El esquema MP posee la ventaja que ellos son muy eficientes al ejecutar. Sin embargo, ellos no incluyen el efecto de nubes. El esquema CC incluye el efecto de nubes pero son costosos computacional.

\section{Metodología}

El ciclo anual de las lluvias en el norte del Perú indica que los máximos acumulados mensuales de lluvias se ubican alrededor de marzo, siendo el periodo de mayores lluvias entre diciembre y abril. El objetivo es medir la predictabilidad de las lluvias en el norte del Perú, se han seleccionado casos de lluvias extrema entre los periodos lluviosos del año 2001 y 2002. En el norte del Perú las lluvias se pueden caracterizar por intensidad y tiempo de duración del evento de lluvia. Así, en una primera aproximación puede diferenciarse tres zonas de precipitación con un grado considerable de homogeneidad: la zona alta de la cuenca $(\mathrm{H})$, la zona media de la cuenca (M) y la zona baja de la cuenca (L). La zona alta de la cuenca la conforman estaciones que se ubican por encima de los $1400 \mathrm{~m}$, la zona media de la cuenca las estaciones que se ubican entre 90 y $1400 \mathrm{~m}$, y la zona baja de la cuenca las estaciones que se ubican debajo de los $50 \mathrm{~m}$. En la presente investigación se han usado 10 estaciones las cuales se indican en la tabla I. La cuarta columna indica a que clasificación pertenece la estación: alta $(\mathrm{H})$, media $(\mathrm{M})$, y baja (L). Los datos fueron proporcionados por el Servicio Nacional de Meteorología (SENAMHI) del Perú y se seleccionaron 14 casos de lluvia extrema. Estos casos serán motivo de evaluación haciendo una comparación con el modelo regional atmosférico.

Tabla I. Ubicación de estaciones de precipitación

\begin{tabular}{|c|c|c|c|c|}
\hline Estación & Lat. & Lon. & Altura & Zona \\
\hline Bernal & -5.63 & -80.75 & 32 & $\mathrm{~L}$ \\
\hline Bigote & -5.28 & -79.70 & 298 & $\mathrm{M}$ \\
\hline Chalaco & -5.03 & -79.83 & 2550 & $\mathrm{H}$ \\
\hline Chulucanas & -5.10 & -80.15 & 95 & $\mathrm{M}$ \\
\hline Chusis & -5.52 & -80.83 & 4 & $\mathrm{~L}$ \\
\hline Huancabamba & -5.25 & -79.45 & 1952 & $\mathrm{H}$ \\
\hline Miraflores & -5.17 & -80.62 & 30 & $\mathrm{~L}$ \\
\hline Morropón & -5.18 & -79.98 & 140 & $\mathrm{M}$ \\
\hline Salpillica & -4.78 & -79.98 & 1456 & $\mathrm{H}$ \\
\hline San Miguel & -5.23 & -80.68 & 29 & $\mathrm{~L}$ \\
\hline
\end{tabular}

Los casos de Lluvias observadas son simulados con el modelo RAMS, en este caso se emplea una configuración estándar. En este experimento se inicializa el modelo con datos de atmósfera del Proyecto Reanálisis de NCEP (National Center for Enviromental Prediction). Además, el acoplamiento usado en este sistema es acoplando la componente oceánica por datos estimado por satélite (AVHRR), climatología durante el periodo 1992-1993, y la componente atmosféricas: vientos, temperatura, altura geopotencial y humedad con datos del proyecto Reanálisis. La simulación presentada tiene dos dominios, uno de resolución de $80 \mathrm{Km}$. con una grilla horizontal de $23 \times 34$ para Perú y otro con una resolución de $20 \mathrm{Km}$. anidado en la zona norte del Perú, con una grilla horizontal de $64 \times 64$, figura 1. En ambos dominios de número de niveles verticales es 30 , con una razón de cambio de "1.23" en cada nivel, de tal manera que en la interfase suelo-atmósfera la densidad de niveles sea mayor. Los eventos de lluvias simulados están definidos en un periodo de 5 días. Así, es posible usar esta información para analizar la predicción de un evento completo de lluvias. La probabilidad que un evento de lluvias en la zona sea de cinco días es menor al 5\% (resultados no publicados). Así, se espera que la simulación de un periodo de cinco días sea suficiente para evaluar un evento completo de precipitación en la zona. Además, esta metodología pretende evaluar la habilidad de la 
configuración del modelo para anticipar el inicio de núcleos convectivos importantes para la formación de lluvia extrema.

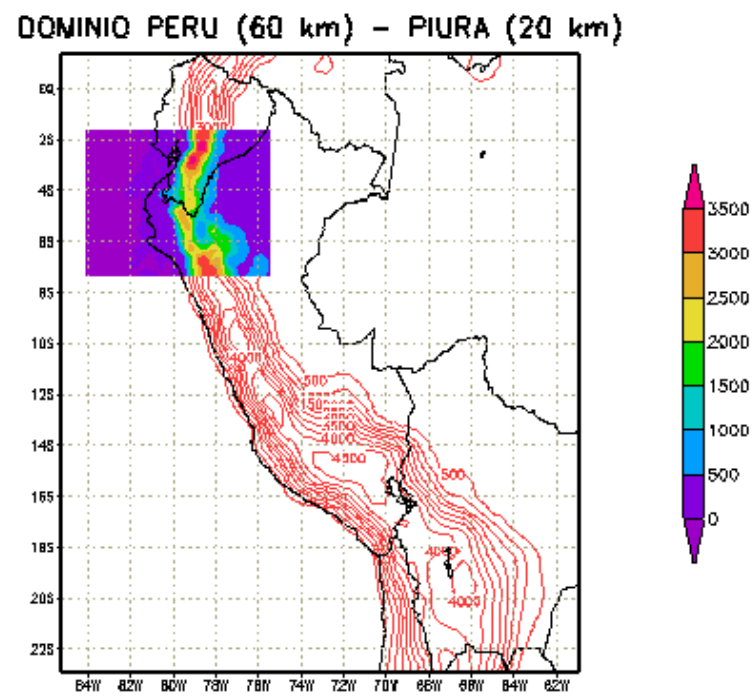

Fig. 1. Dominio grueso y anidado usado en la simulación. El color sombreado representa el dominio para la zona norte del Perú, el cual tiene una resolución de 20 km., el dominio grande tiene una resolución de $80 \mathrm{~km}$.

\section{Análisis}

Los eventos de lluvias seleccionados son 14 , los cuales se indican a continuación. Durante el año 2001: 15 de enero, 18 y 24 febrero, 13,15 y 24 marzo, 10 y 30 diciembre; y durante el año 2002: 2 y 25 febrero, 2,16 y 25 marzo, y 3 de diciembre. En la figura 2 se presenta la secuencia de lluvias observadas en la zona de estudios para el caso del 25 de febrero del 2002. Este evento de lluvias se inicia del 25 de febrero y finaliza el 29 de febrero. Se puede observar que la máxima lluvias se presenta el día 28 de febrero. La lluvia máxima observada es de 75 $\mathrm{mm}$ y corresponde a una estación en la cuenca media (M). En la figura 3 se presenta la secuencia de lluvias simuladas por el modelo RAMS en la zona de estudio para el mismo caso del 25 de febrero del 2002. La secuencia corresponde a los días durante el 25 y 29 de febrero. Se observa que la máxima cantidad de lluvias se observa también el día 28 de febrero. Se puede observar un núcleo de precipitación considerable alrededor de la latitud $-5.5^{\circ}$ y la longitud $-80.0^{\circ}$, este núcleo coincide con el núcleo en los valores observado. Otros patrones no pueden verificarse debido a que las estaciones observadas miden lugares geográficos puntuales (10 en el presente estudio).
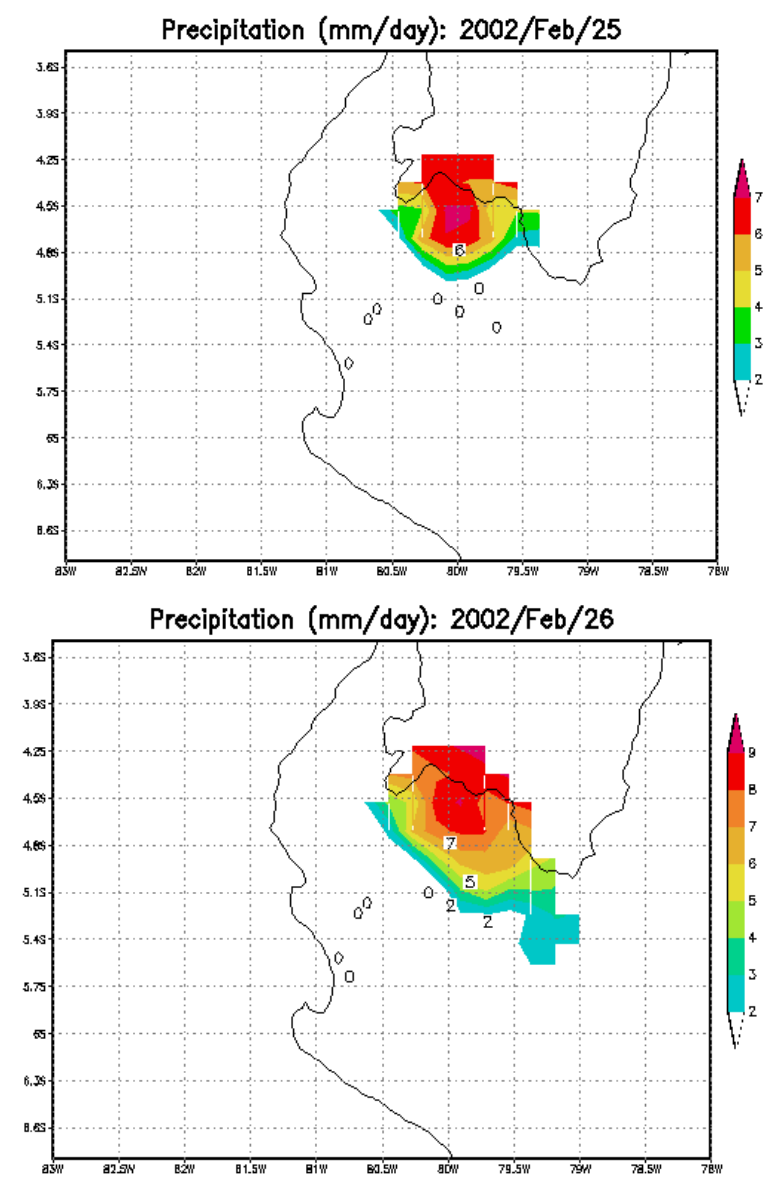

Precipitation (mm/day): 2002/Feb/27

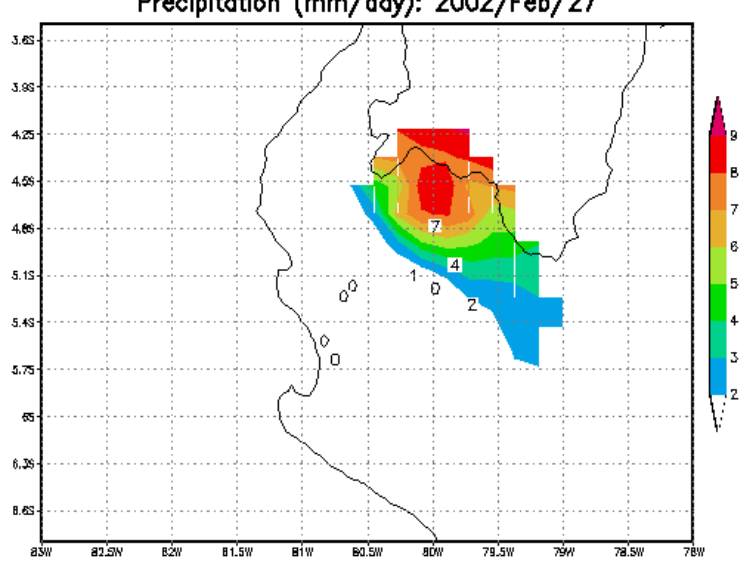

Fig. 2. Evolución temporal y espacial de precipitación observada en el área de estudio. Caso: 25 de febrero de 2002. 

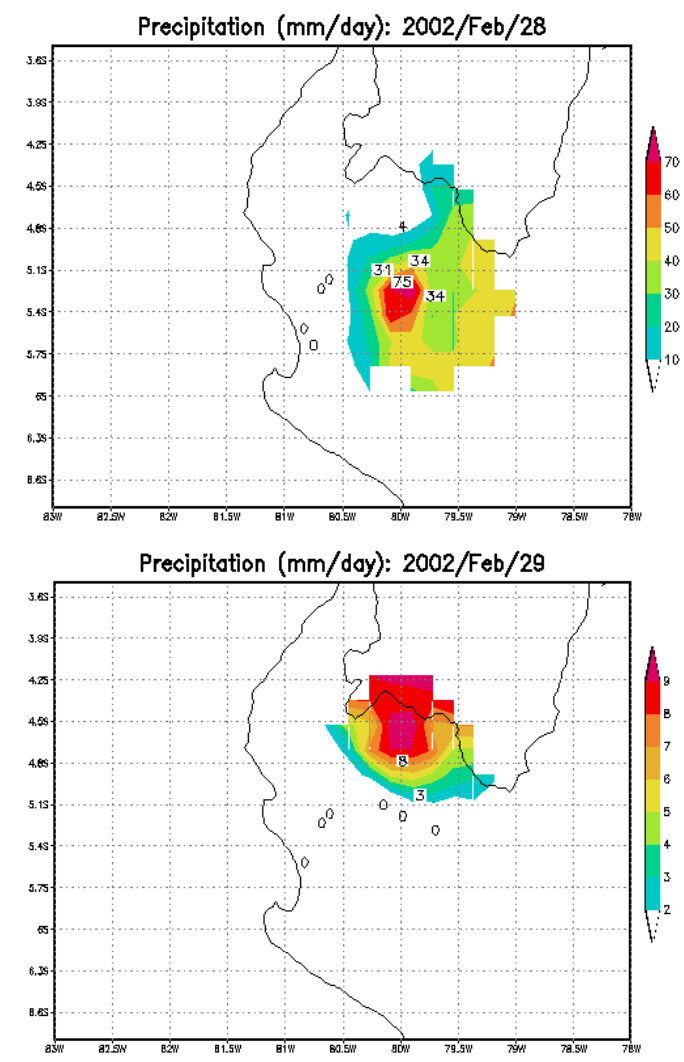

Fig. 2. Continuación.

En este caso del 25 de febrero la simulación es óptima. Lo descrito visualmente puede cuantificarse con el siguiente resultado: correlación de 0.85 para la zona alta, 0.94 para la zona media, y 0.00 para la zona baja. Otro caso a evaluar es del 16 de marzo de 2006, en la figura 4 se presenta una serie de tiempo de la precipitación para la zona alta, media, y baja. Las correlaciones para este caso son: 0.94, 0.98 y 0.40 para la zona alta, media y baja respectivamente. Análogamente al caso anterior la mayor correlación se presento en la zona media. El bajo nivel de correlación en la zona baja se debe a casos nulos de lluvias observadas. Este valor siempre será muy bajo porque las lluvias en la zona baja tienen frecuencia de un día. Un análisis de frecuencia de precipitación diaria en la zona verifica que la probabilidad de ocurrencia de lluvias dado que el día anterior hubo un registro de lluvias es 0.10 .
[25Feb02] Precipitation $(\mathrm{mm} /$ day): 25Feb02

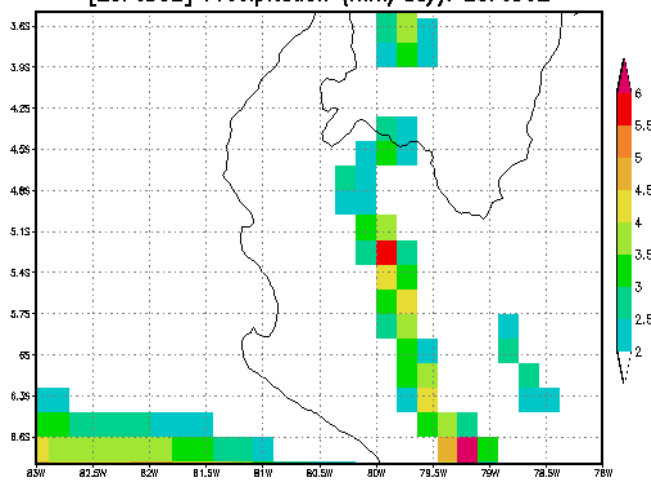

SIMULACION:

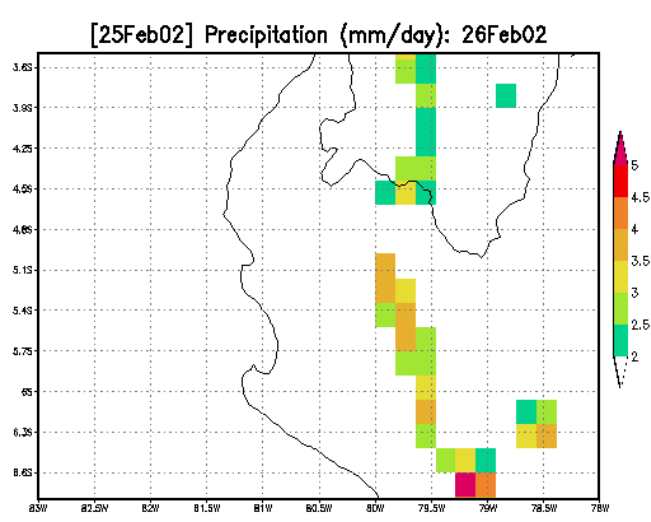

[25Feb02] Precipitation $(\mathrm{mm} / \mathrm{day}): 27 \mathrm{Feb02}$

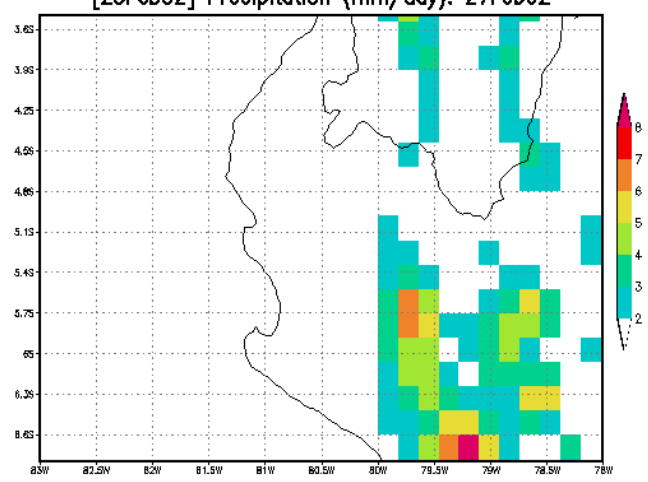

[25Feb02] Precipitation $(\mathrm{mm} /$ day): $28 \mathrm{Feb02}$

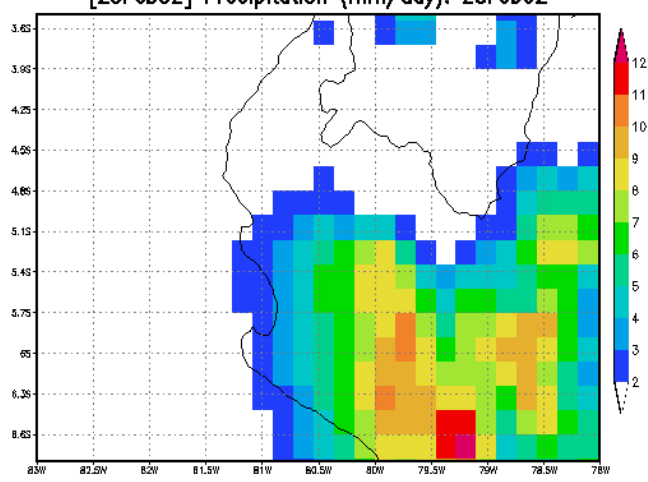

Fig. 3. Evolución temporal y espacial de precipitación simulada en el área de estudio. Caso: 25 de febrero de 2002. 


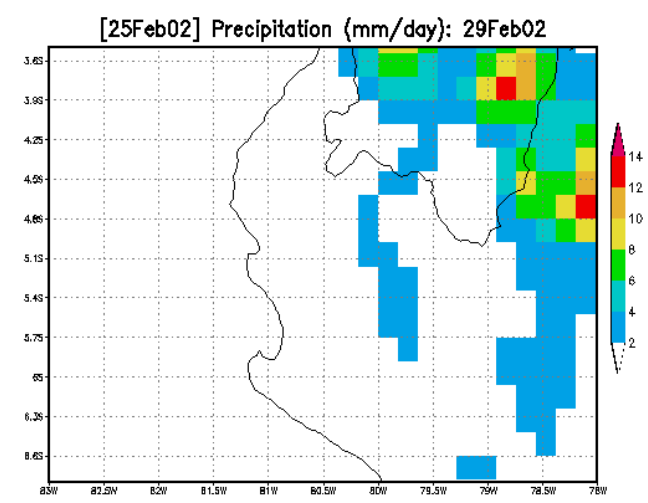

Fig. 3. Continuación.
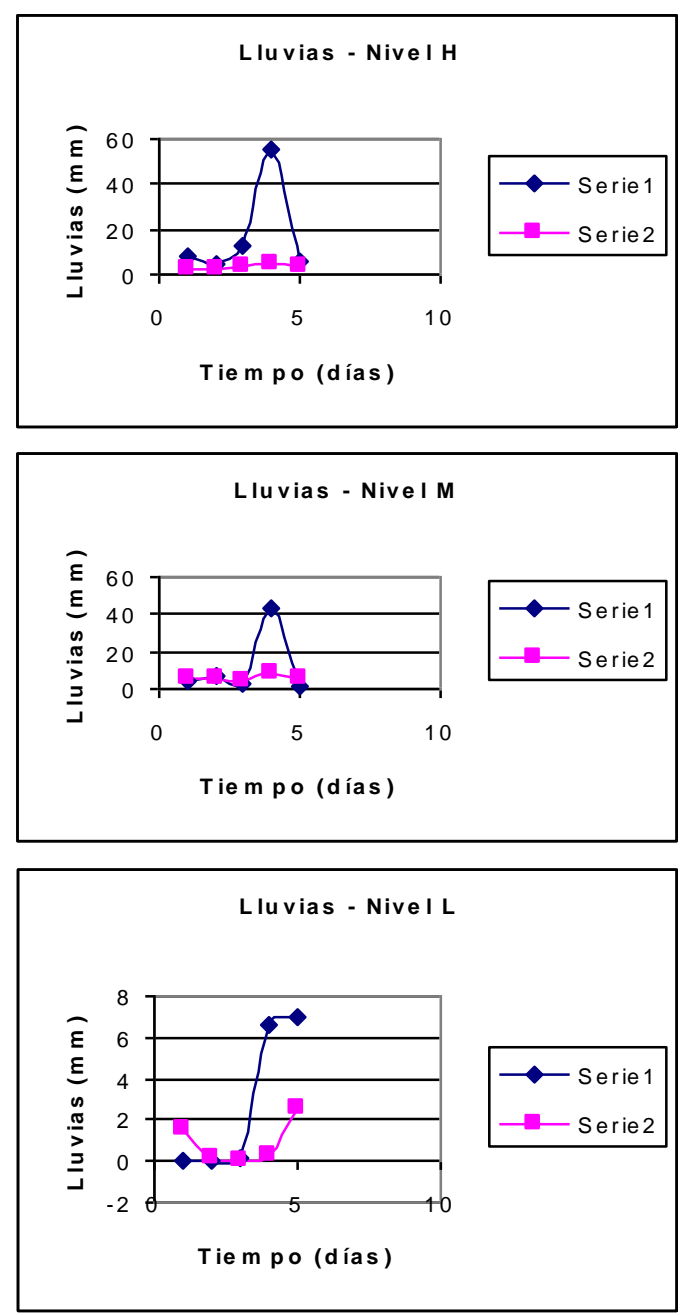

Fig. 4. Serie de tiempo de un caso de lluvia extrema según las zonas de interés.

Por ello se puede afirmar que se tendrá muchos valores nulos observados en la zona baja. Es importante mejorar las condiciones de contorno (Temperatura Superficial del Mar, TSM) ingestadas por el modelo para corregir estos valores de precipitación en la zona baja. Como se puede observar en la figura 4 , los valores simulados de lluvias no están normalizados a los valores observados. Lo importante es mostrar que las simulaciones logran evaluar los picos de precipitación, información importante para la gestión de sistemas de alerta. La solución a una cuantificación de valores de precipitación simuladas por el modelo RAMS se puede observar en la figura 5, allí una regresión lineal de la lluvia simulada versus la pronosticada se presenta, esto permite transformar valores de modelo a valores de lluvias reales.

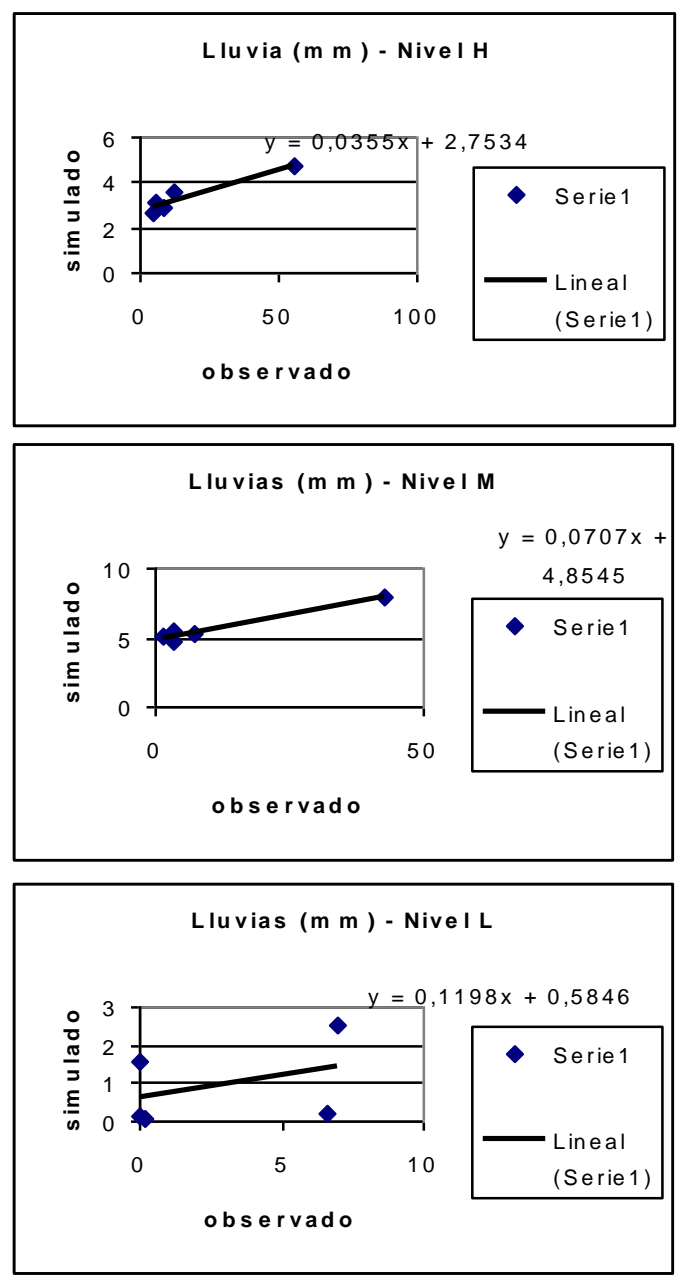

Fig. 5. Regresión lineal de un caso de lluvia extrema según las zonas de interés.

Las tres zonas presentan diferentes valores de pendiente, la zona $\mathrm{H}$ y $\mathrm{M}$ tienen un vació de datos entre 20 y $40 \mathrm{~mm}$. El valor pico que esta entre 40 y $50 \mathrm{~mm}$ para la zona $\mathrm{H}$ y $\mathrm{M}$ se presenta 
en el día 4 de simulación. Esta lluvia extrema es simulada cuatro días antes de su ocurrencia para la zona $\mathrm{H}$ y $\mathrm{M}$; según la figura 4c la simulación máxima la ubica el día 5 después de iniciada la simulación.

Este desfase puede deberse a problemas costeros en los mecanismos de interacción océano-tierra. Debe recordarse que los valores de TSM (Temperatura superficial del Mar) son tomados de valores climáticos. Valores "reales" para los días de simulación pueden mejorar los resultados de correlación en la zona baja de estudio.

Tabla II. Correlación (r) de eventos de lluvias para la zona baja. (Promedio Mensual - P.M.)

\begin{tabular}{|c|c|c|c|c|c|}
\hline \multicolumn{7}{|c|}{ NIVEL : L } \\
\hline Fecha & $\mathbf{r}$ & Fecha & $\mathbf{r}$ & P.M. & \\
\hline $15-01-01$ & 0.80 & $10-12-01$ & 0.00 & & \\
\hline $18-02-01$ & 0.00 & $30-12-01$ & 0.00 & & \\
\hline $24-02-01$ & 0.69 & $03-12-02$ & -0.29 & -0.09 & Dic \\
\hline $13-03-01$ & -0.23 & $15-01-01$ & 0.80 & 0.80 & Ene \\
\hline $15-03-01$ & -0.48 & $18-02-01$ & 0.00 & & \\
\hline $24-03-01$ & 0.90 & $24-02-01$ & 0.69 & & \\
\hline $10-12-01$ & 0.00 & $02-02-02$ & 0.52 & & \\
\hline $30-12-01$ & 0.00 & $25-02-02$ & 0.00 & 0.30 & Feb \\
\hline $02-02-02$ & 0.52 & $13-03-01$ & -0.23 & & \\
\hline $25-02-02$ & 0.00 & $15-03-01$ & -0.48 & & \\
\hline $02-03-02$ & -0.36 & $24-03-01$ & 0.90 & & \\
\hline $16-03-02$ & 0.40 & $02-03-02$ & -0.36 & & \\
\hline $25-03-02$ & 0.16 & $16-03-02$ & 0.40 & & \\
\hline $03-12-02$ & -0.29 & $25-03-02$ & 0.16 & 0.06 & Mar \\
\hline Prom. & $\mathbf{0 . 1 5}$ & & & & \\
\hline
\end{tabular}

Finalmente, se presenta las tablas II, III, y IV con una descripción de las correlaciones de todos los eventos seleccionados. Estos resultados están divididos por niveles $(\mathrm{H}, \mathrm{M}, \mathrm{y} \mathrm{L})$. Además un promedio global es presentado. Así, la mayor correlación se observa en la parte alta de zona con un valor de 0.489 , la correlación para la zona media es 0.436 , y el menor valor de correlación promedio en la zona baja con 0.150 . Adicionalmente, se presentan valores promedios de correlación mensual.
Tabla III. Correlación (r) de eventos de lluvias para la zona media. (Promedio Mensual - P.M.)

\begin{tabular}{|c|c|c|c|c|c|}
\hline \multicolumn{7}{|c|}{ NIVEL : M } \\
\hline Fecha & $\mathbf{r}$ & Fecha & $\mathbf{r}$ & P.M. & \\
\hline $15-01-01$ & 0.67 & $10-12-01$ & 0.00 & & \\
\hline $18-02-01$ & 0.71 & $30-12-01$ & 0.00 & & \\
\hline $24-02-01$ & -0.04 & $03-12-02$ & 0.00 & 0.00 & Dic \\
\hline $13-03-01$ & 0.76 & $15-01-01$ & 0.67 & 0.67 & Ene \\
\hline $15-03-01$ & 0.15 & $18-02-01$ & 0.71 & & \\
\hline $24-03-01$ & 0.22 & $24-02-01$ & -0.04 & & \\
\hline $10-12-01$ & 0.00 & $02-02-02$ & 0.84 & & \\
\hline $30-12-01$ & 0.00 & $25-02-02$ & 0.94 & 0.61 & Feb \\
\hline $02-02-02$ & 0.84 & $13-03-01$ & 0.76 & & \\
\hline $25-02-02$ & 0.94 & $15-03-01$ & 0.15 & & \\
\hline $02-03-02$ & 0.84 & $24-03-01$ & 0.22 & & \\
\hline $16-03-02$ & 0.98 & $02-03-02$ & 0.84 & & \\
\hline $25-03-02$ & 0.00 & $16-03-02$ & 0.98 & & \\
\hline $03-12-02$ & 0.00 & $25-03-02$ & 0.00 & 0.49 & Mar \\
\hline Prom. & $\mathbf{0 . 4 3}$ & & & & \\
\hline
\end{tabular}

Donde para la zona media, febrero (0.61) presenta mejores correlaciones respecto a marzo (0.49), y enero (0.67) mejores valores de correlación respecto a febrero (0.61). Este último caso puede ser poco relevante, pues solo un caso de lluvia en enero fue evaluado en las dos estaciones lluviosas (2001-2002). Para la zona alta las relaciones son similares y también para la zona baja.

Tabla IV. Correlación (r) de eventos de lluvias para la zona alta. (Promedio Mensual - P.M.)

\begin{tabular}{|c|c|c|c|c|c|}
\hline \multicolumn{7}{|c|}{ NIVEL : H } \\
\hline Fecha & $\mathbf{r}$ & Fecha & $\mathbf{r}$ & P.M. & \\
\hline $15-01-01$ & 0.70 & $10-12-01$ & 0.30 & & \\
\hline $18-02-01$ & 0.96 & $30-12-01$ & -0.27 & & \\
\hline $24-02-01$ & 0.04 & $03-12-02$ & 0.87 & 0.30 & Dic \\
\hline $13-03-01$ & 0.59 & $15-01-01$ & 0.70 & 0.70 & Ene \\
\hline $15-03-01$ & 0.84 & $18-02-01$ & 0.96 & & \\
\hline $24-03-01$ & 0.92 & $24-02-01$ & 0.04 & & \\
\hline $10-12-01$ & 0.30 & $02-02-02$ & 0.85 & & \\
\hline $30-12-01$ & -0.27 & $25-02-02$ & 0.85 & 0.68 & Feb \\
\hline $02-02-02$ & 0.85 & $13-03-01$ & 0.59 & & \\
\hline $25-02-02$ & 0.85 & $15-03-01$ & 0.84 & & \\
\hline $02-03-02$ & 0.11 & $24-03-01$ & 0.92 & & \\
\hline $16-03-02$ & 0.94 & $02-03-02$ & 0.11 & & \\
\hline $25-03-02$ & -0.90 & $16-03-02$ & 0.94 & & \\
\hline $03-12-02$ & 0.87 & $25-03-02$ & -0.90 & 0.41 & Mar \\
\hline Prom. & $\mathbf{0 . 4 8}$ & & & & \\
\hline
\end{tabular}


Aun cuando las correlaciones en promedio son bajas, debe resaltarse las correlaciones de 0.90 , 0.94 , y 0.96 encontradas en casos individuales. Es importante evaluar las condiciones a escala sinóptica que hacen diferentes la habilidad del modelo RAMS a reproducir las condiciones de lluvias en tiempo extremo.

\section{Conclusiones}

El modelo regional usado ha mostrado una gran habilidad para diagnosticar los casos de lluvia extrema en la zona de estudio. Correlaciones del orden de 0.90 para casos evaluados lo confirman. La mayor correlación se encuentra en la zona alta, seguida por la media, y finalmente la zona baja. Esto es un resultado importante pues la información crucial para el diseño de alerta temprana son las precipitaciones en la parte alta de la cuenca. Para el caso del 16 de marzo de 2002 el modelo RAMS ha mostrado que puede diagnosticar el pico de lluvia extrema cuatro días antes de ocurrido el evento. Los bajos valores de correlación en la zona baja se deben posiblemente a los procesos costeros de interacción aire-océano. Una evaluación con datos reales en lugar de climatológicos pueden dar mejores resultados. Esto no es critico debido a que la lluvia extrema se genera en la zona alta y media.

\section{Agradecimientos}

Queremos agradecer al Servicio Nacional de Meteorología e Hidrología del Perú (SENAMHI) por su apoyo en esta investigación, su soporte computacional y el uso de su información. A la Universidad Nacional Mayor de San Marcos, especialmente al Consejo Superior de Investigación por patrocinar y apoyar la realización de la presente investigación. A la NCEP por el acceso a sus datos del proyecto Reanálisis. Y un agradecimiento especial al FENLAB de la UNMSM por el tiempo invertido en las discusiones.

\section{Referencias}

[1] Horel, J. D., y Cornejo-Garrido, A. G., (1986). Mon. Weat. Rev., 114, 2091-2105.

[2] Bendix, J., (2000). Int. J. Remote Sensing, vol. 21 , No. $3,533-548$.

[3] Tripoli, G.J. and W.R.Cotton., J. de Rech. Atmos., 16, (1982) 185-195.

[4] Tremback, C.J., Ph.D. dissertation, (1990) Colorado State University.

[5] Mahrer, Y. and R.A. Pielke, Contrib. Atmos. Phys., 50, (1977) 98-113.

[6] Mesinger, F. and A. Arakawa, GARP Publication Series, No. 14, WMO/ICSU Joint Organizing Committee, (1976) 64 pp.

[7] Gal-Chen, T., and R.C.J. Somerville, J. Comput. Phys., 17, (1975) 209-228.

[8] Clark, T.L., and R.D. Farley, J. Atmos. Sci.,41, (1984) 350.

[9] Clark, T.L., and W.D. Hall, J. Comput. Phys., 92, (1991) 456-481.

[10] Tremback, C.J., and R. Kessler, Preprints, 7th Conference on Numerical Weather Prediction, 17-20 June 1985, Montreal, Canada, (1985) AMS.

[11] Louis, J.F., Boundary-Layer Meteorol.,17, (1979) 187-202.

[12] Businger, J.A., J.C. Wyngaard, Y. Izumi, and E.F. Bradley, J. Atmos. Sci., 28, (1971) 181-189.

[13] Avissar, R. and R.A. Pielke, Mon. Wea. Rev., 117, (1989) 2113-2136.

[14] Kuo, H.L., J. Atmos. Sci., 35, (1974) 12321240.

[15] Chen, S. and W.R. Cotton, J. Atmos. Sci., 45, (1988) 3897-3910. 